\title{
Screening and identification of peritoneal metastasis-related genes of gastric adenocarcinoma using a cDNA microarray
}

F.H. Bai ${ }^{1,2 *}$, N.J. Wang ${ }^{3 *}$, J. Wang ${ }^{1 *}$, L. Yang ${ }^{2}$, F.M. Zhang ${ }^{1}$, F. Yin ${ }^{1}$, J. Liang ${ }^{1}$, K.C. Wu ${ }^{1}$ and D.M. Fan ${ }^{1}$

${ }^{1}$ State Key Laboratory of Cancer Biology and Institute of Digestive Diseases, XiJing Hospital, Fourth Military Medical University, Xi'an, Shaanxi, China ${ }^{2}$ Department of Gastroenterology, Affiliated Hospital of Ninxia Medical College, Yinchuan, China

${ }^{3}$ Department of Oncology, Affiliated Hospital of Ninxia Medical College, Yinchuan, China

*These authors contributed equally to this study.

Corresponding author: D.M. Fan

E-mail: fandaim@yeah.net

Genet. Mol. Res. 11 (2): 1682-1689 (2012)

Received June 28, 2011

Accepted January 5, 2012

Published June 25, 2012

DOI http://dx.doi.org/10.4238/2012.June.25.1

\begin{abstract}
With the aim of identifying peritoneal metastasis-related genes in gastric cancer, we performed a broad analysis of differential gene expression between the parental cell line GC9811 and its highly metastatic peritoneal counterpart, cell line GC9811-P. Two fluorescent cDNA probes, labeled with $\mathrm{Cy} 3$ and $\mathrm{Cy} 5$ dyes, were prepared from GC9811 and GC9811-P mRNA samples by the reverse transcription method. The two color probes were then mixed and hybridized to a cDNA chip constructed with double-dots from 11,901 human genes; this was scanned at two wavelengths. The experiment was repeated twice. In GC9811-P cells, 218 genes were upregulated and 30 genes were downregulated compared with the parental cell lines. Some selected genes were confirmed by RT-PCR and Western blot; we found that S100A4 and CTNNB1 were upregulated
\end{abstract}


and PTEN was downregulated in GC9811-P cells. Identification of these differentially expressed genes could contribute to disclose the molecular mechanisms involved and provide new targets for therapeutic intervention to avoid peritoneal dissemination of gastric adenocarcinoma.

Key words: Gastric cancer; Peritoneal dissemination; cDNA microarray; Differential gene expression

\section{INTRODUCTION}

Peritoneal dissemination is the most frequent type of recurrence in advanced gastric cancer (Jemal et al., 2005; Yoshikawa et al., 2006; Yonemura et al., 2007), and the prognosis for dissemination is the worst among the recurrence patterns of gastric cancer, with a mean survival of 6 months. Previous research indicated that peritoneal dissemination is required in many steps of the complex metastatic process, including the interaction of many genes along with their products, self-regulation, and interference with multiple signal transduction pathways (Yanagihara et al., 2005; Motoori et al., 2006). However, little is known about the details of multiple genetic alterations in peritoneal dissemination of gastric cancer. Although several molecules have been reported to be involved in the peritoneal metastasis of gastric cancer, it is known that a single or even a few selected molecules cannot define the whole biological characteristics of a tumor. Thus, systematic analysis of gene expression profiling of peritoneal dissemination is essential.

To investigate the mechanism of peritoneal dissemination in gastric cancer, we have established a cell line, GC9811-P, with a high potential for peritoneal metastasis of gastric cancer compared to its parental cell line GC9811 (Bai et al., 2007). However, its common changes in gene expression have not yet been clarified. In the present study, a cDNA microarray system was used to screen high-risk genes for peritoneal metastasis in gastric cancer.

\section{MATERIAL AND METHODS}

\section{Cell culture and RNA preparation}

GC9811 and GC9811-P cells were grown in Dulbecco's modified Eagle's medium (DMEM) containing $10 \%$ fetal bovine serum (FBS), in $5 \% \mathrm{CO}_{2}$ at $37^{\circ} \mathrm{C}$. When they reached $80-90 \%$ confluence, cells were washed with ice-cold PBS and homogenized immediately. Total RNA was extracted from each cell line according to the original single-step extraction procedure, which came with the RNA kit (Gibco, USA), with a slight modification.

\section{Construction of the microarray}

The construction of cDNA microarrays was carried out and processed essentially following previous study (Schieren et al., 2006). The microarray sequences included 12,800 full-length and partial complementary DNAs (cDNAs) representing known, novel, and control genes, provided by Lifegen, Co., Shannxi, China. The cDNA inserts were amplified by polymerase chain reaction (PCR) using universal primer sequences and then purified. The PCR products were then spotted onto siliconized slides (CEL Associates, Houston, TX, USA) with Cartesian Pix Sys 7500 
spotting robotics (Cartesian Technologies, Irvine, CA, USA). The slides were subsequently hydrated, ultraviolet cross-linked, and soaked. Finally, the slides were dried again and ready for use.

\section{Probe preparation}

Messenger RNA (mRNA) was purified using an Oligotex-dt mRNA Midi kit (Qiagen, Carlsbad, CA, USA). The fluorescent cDNA probes were prepared by reverse transcription and then purified according to Schena et al. (1995). The RNA samples from the GC9811 cells were labeled with cyanogen (Cy) 3-deoxyuridine triphosphate (Cy3-dUTP) and those from the GC9811-P cells with Cy5-dUTP. The two color probes were precipitated with ethanol, and dissolved in $20 \mu \mathrm{L}$ hybridization solution (5X SSC, $0.4 \%$ SDS, 50\% formamide, 5X Denhardt's solutions).

\section{Hybridization and washing}

The chips were prehybridized with the hybridization solution containing $0.5 \mathrm{mg} / \mathrm{mL}$ denatured salmon sperm DNA at $42^{\circ} \mathrm{C}$ for $6 \mathrm{~h}$. Fluorescent probe mixtures were denatured at $95^{\circ} \mathrm{C}$ for $5 \mathrm{~min}$, and the denatured probe mixture was added to the pre-hybridized chip under a cover glass. The chips were incubated at $42^{\circ} \mathrm{C}$ for 15 to $17 \mathrm{~h}$ and washed in solutions of $2 \mathrm{X} \mathrm{SSC}+0.4 \% \mathrm{SDS}$, $0.1 \mathrm{X} \mathrm{SSC}+0.4 \% \mathrm{SDS}$, and $0.1 \mathrm{X} \mathrm{SSC}$ at $60^{\circ} \mathrm{C}$ each for $10 \mathrm{~min}$, then dried at room temperature.

\section{Detection and analysis}

The chips were scanned with an Axon 4000A scanner (Axon Instruments Inc., Union City, CA, USA). At two wavelengths to detect emissions from both $\mathrm{Cy} 3$ and $\mathrm{Cy} 5$, the acquired images were analyzed using the GenePix 3.0 software. The log-ratios of fluorescence intensities for $\mathrm{Cy} 3$ or Cy5 were computed for each location on each microarray. Overall intensities were normalized by the Probe Set Normalization method. Genes were identified as differentially expressed if the absolute value of the natural logarithm of the ratio was $>0.69$. To minimize artifacts arising from low expression values, only Cy 3 and Cy5 with counts $>1000$ and ratios $>2$ or $<0.5$ were chosen for further analysis.

\section{RT-PCR and Western blotting}

Some of the selected genes were further studied. Semi-quantitative RT-PCR and Western blot analysis were used to validate the microarray results. Semi-quantitative RT-PCR was performed using a routine method. Western blot analysis was carried out as described previously (Wang et al., 2006).

\section{RESULTS}

\section{Microarray manufacture and testing}

Microarrays were constructed containing a total of 11,901 genes or 12,800 spots per chip. These chips contained most of the cDNAs of known sequence and function, some novel cDNAs not present in public databases, and control spots of non-human origin. Hybridization 
experiments with probes prepared from human mRNA produced little or no signal at these positions in the negative controls and strong signal in the positive controls (data not shown), which confirms that our data are reliable. All results were representative of two independent experiments and each gene was spotted with two dots. Therefore, false-positives were reduced to approximately $0.09 \%(3 \times 3 \%)$ in the two separate experiments, and the differentially expressed genes between the GC9811 and GC9811-P cells at this level were not a significant source of false-positives.

\section{Differential gene expression between GC9811 and GC9811-P cells}

We performed a global analysis of gene expression of 11,901 genes in GC9811 and GC981-P cells using microarray. In comparison to GC9811 cells, 248 of the total genes $(0.02 \%)$ revealed differential expression in repeated experiments, 218 genes (ratios $>2.0$ ) revealed elevated expression, and 30 genes (ratios $<0.5$ ) revealed reduced expression in GC9811-P cells. Consistent with the assay, scatter plots comparing the results of GC9811 cells to GC9811-P cells showed an even distribution of ratios (Figure 1). The top 10 upregulated and top 10 downregulated differentially expressed genes in the peritoneum metastatic subline GC9811-P compared to the parental cell line GC9811 are shown in Table 1.

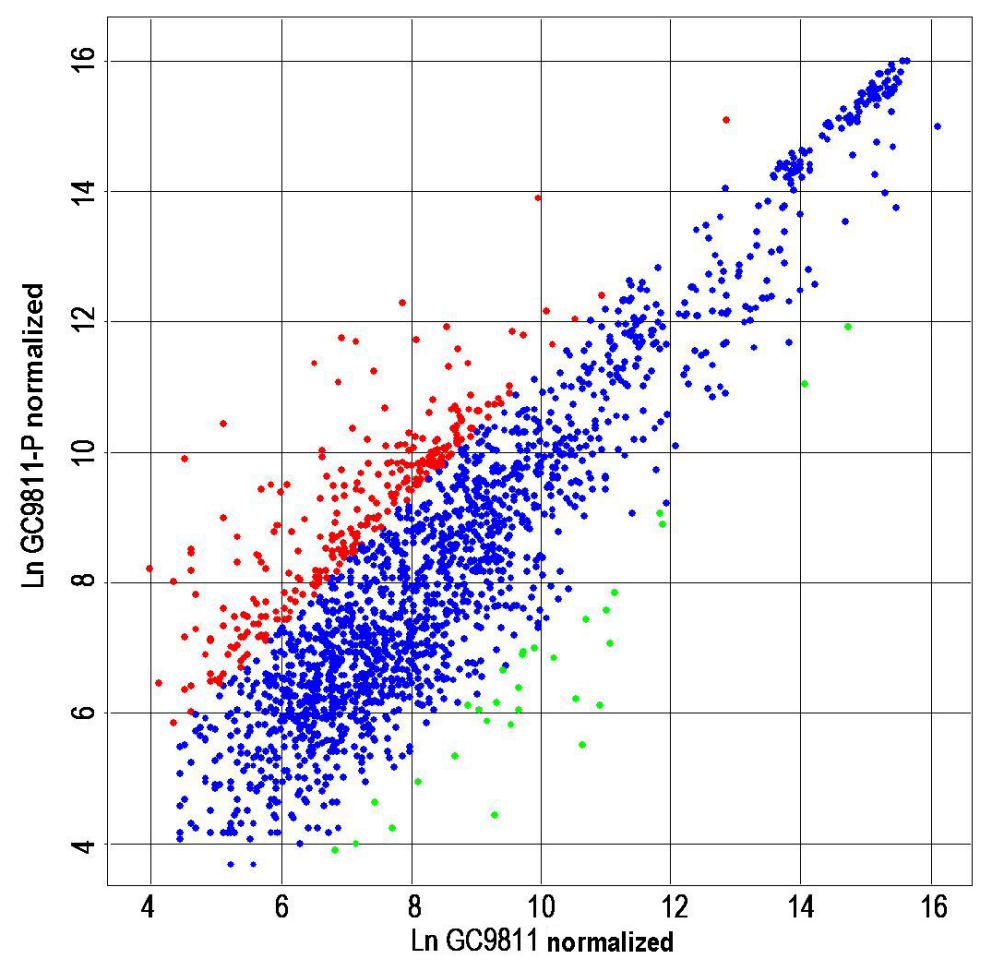

Figure 1. Microarray scatter plots. Blue spots indicate that the ratios of expression values are between 0.5 and 2.0, showing no differential expression of the gene between GC9811 and GC9811-P cells. Red spots or green spots indicate that the ratios of expression values are below 0.5 or above 2.0 , demonstrating differential expression of the genes between GC9811 and GC9811-P cells. 
Table 1. Interesting differentially expressed genes in GC9811-P vs GC9811 cells.

\begin{tabular}{llll}
\hline GenBank & Gene name & Function & Ratio \\
\hline Upregulated genes & & & \\
NM_002961 & S100A4 & Invasion and metastasis & 153.7037 \\
AF024617 & ZNRD1 & Mutidrug resistant & 2.421652 \\
U28368 & ID4 & DNA synthesis and repair & 2.106028 \\
NM_022661 & SPANXC & Spermiogenesis & 74.53704 \\
X66899 & EWSR1 & Oncogene, transcriptional activation & 3.762095 \\
M63509 & GSTM2 & Metabolism & 20.37037 \\
U23803 & HNRPA0 & Protein synthesis and modification & 67.77778 \\
NM_003807 & TNFSF14 & Related to apoptosis & 2.136752 \\
NM_016932 & SIX2 & Development & 33.64198 \\
X87838 & CTNNB1 & Adhesion & 5.092593 \\
Downregulated genes & & & 0.028578 \\
U96180 & PTEN & Tumor suppressor \\
D32050 & AARS & Metabolism & 0.001693 \\
U37143 & CYP2J2 & Metabolism & 0.007123 \\
NM_014255 & TMEM4 & Transmembrane protein & 0.006759 \\
NM_007259 & VPS45B & Vacuolar protein sorting 45B & 0.005511 \\
M_014424 & HSPB7 & Heat shock 27-kDa protein family & 0.063311 \\
M59818 & CSF3R & Colony-stimulating factor 3 receptor & 0.05352 \\
NM_006901 & MYO9A & Cystoskeleton and motility & 0.027233 \\
NM_013363 & PCOLCE2 & Procollagen C-endopeptidase enhancer 2 \\
NM_013370 & OKL38 & Pregnancy-induced growth inhibitor & \\
NM_020179 & FN5 & Transcription factor & 0.002356 \\
\hline & & & 0.102881 \\
& & & 0.16392 \\
\hline
\end{tabular}

\section{Complete concordance of differentially expressed genes by RT-PCR and Western blot}

To validate the differential gene expression between GC9811 cells and GC9811-P cells, we studied partial selected genes by semi-quantitative RT-PCR and Western blot. Selected genes identified as being upregulated differentially expressed, S100A4 protein and catenin beta-1 (CTNNB1), were found in the highly metastatic cells, and phosphatase and tensin homolog deleted on chromosome ten (PTEN) was found to be downregulated in the metastatic cell line, as had been determined using microarrays. The results of both RT-PCR (Figure 2A) and Western blotting (Figure 2B) were completely concordant with the array analysis.

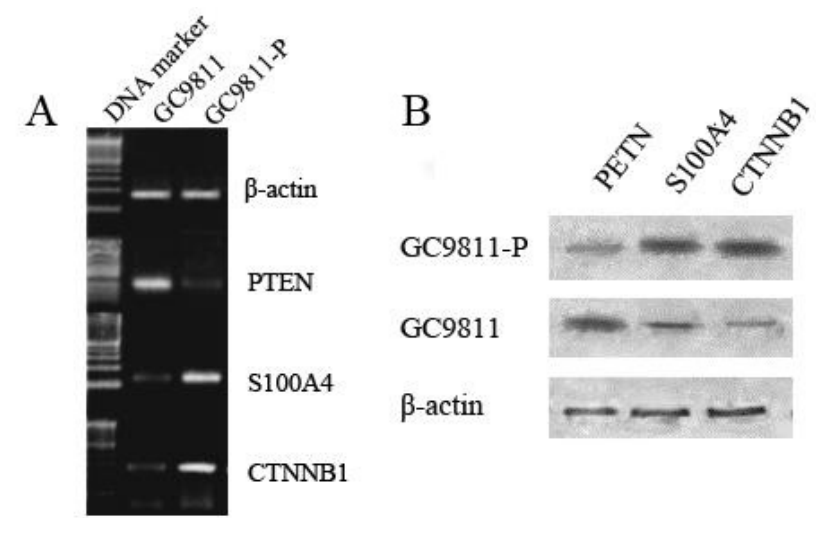

Figure 2. Results of semi-quantitative RT-PCR (A) and Western blotting (B) analysis of PTEN, S100A4, CTNNB1 expression in high-metastatic subline cells (GC9811-P) and parental cell lines (GC9811). The expression of S100A4 and CTNNB1 was elevated in high-metastatic subline cells (GC9811-P) vs parental cell lines (GC9811), and PTEN expression was downregulated in high-metastatic subline cells (GC9811-P) vs parental cell lines (GC9811). $\beta$-actin was shown as a loading control. 


\section{DISCUSSION}

To identify gastric carcinoma with peritoneal metastasis-related genes, we utilized the GC9811 cell line and GC9811-P cell line as a pair in the model. Screening of the differentially expressed genes under such small different genetic background displays could remove the irrelevant difference. Therefore, the result with the interesting genes such as peritoneal-metastatic potential can be identified and more reliably. In our study, the relative mRNA levels of genes expressed in the cell lines were identified using cDNA microarrays. The results suggested that 218 genes were upregulated and 30 genes were significantly downregulated. Consistent with the assay, the result of scatter plots showed an even distribution of ratios, suggesting that the differences between the differentially expressed genes of the GC9811 cells to GC9811-P cells were not so large.

The malignant features of cancer cells such as adhesion, invasion, motility, migration, metabolism, resistance to apoptosis, and cell cycle abnormalities play a pivotal role in peritoneal dissemination. Several different expression genes between the primary gastric cancer cell lines and its high peritoneal metastasis subline have been previously reported to be related to these features (Hippo et al., 2001; Mori et al., 2004; Yanagihara et al., 2005). The other genes from our results that probably involved peritoneal dissemination of gastric cancers have not yet been reported and the precise function of these genes remain unclear, so that further studies would be necessary to clarify this.

Some of the genes such as S100A4 and heat shock protein 40 (HSP40) that were upregulated reflected the distinct characteristics of peritoneal metastasis of gastric cancer, and they were also found to be upregulated in our study. The S100A4 gene is a member of the S100 protein family and has functions in the cell cycle and cell motility. Previous papers reported that several cancers, such as breast cancer, colon cancer, and osteosarcoma, were known to produce S100A4, which is closely related to tumor aggressiveness and metastasis (Yonemura et al., 2000; Cho et al., 2003). Hsp40 facilitates the critical role of Hsp70 in a number of cellular processes such as protein folding, assembly, degradation, and translocation in vivo ( $\mathrm{Li}$ et al., 2006). The previous study indicated that the HSP40 gene was correlated with tumor development and metastasis (Motoori et al., 2006). It is well known that the alteration in adhesion molecule-related genes facilitates cell escape from the primary site, and finally leading to metastasis (Arboleda et al., 2003; Motoori et al., 2006). Our data showed increased expression of cell adhesion genes, such as CTNNB1, PXN and CDH13, and were correlated with increased invasion and metastasis. Cell cycle control-related genes, such as GSPT1 and CKS1, were also found to be involved in invasion and metastasis of malignant tumor (Huerta et al., 2003; Brito et al., 2005). Our findings suggest that GSPT1 and CKS1 were upregulated in GC9811-P cells, which may contribute to peritoneal invasive or metastatic processes in gastric adenocarcinoma. The invasive ability of GC9811-P cells triggered by GSPT1 and CKS1 may involve an unknown mechanism that regulates the expression of one of its downstream target genes. In previous studies, the positive rate of PTEN was significantly lower in gastric cancer than in normal gastric tissue and distant metastasis (Li et al., 2007). Low expression of the product of the PTEN gene was correlated with the depth of invasion and the presence of metastases in gastric cancer (Lazăr et al., 2006). Expression of S100A4 in gastric cancer is significantly associated with lymph node and distant metastases and poor prognosis (Wang et al., 2010). The positive expression of S100A4 was significantly associated with advanced gastric cancer, higher pTNM stage, and poorer survival rates, especially when present in nuclear 
staining (Yoon et al., 2008). Loss of membrane/cytoplasmic $\beta$-catenin expression in the tumor center correlated with pT, loss at the invasion front with pTNM stage (Retterspitz et al., 2010). $\beta$-catenin immunoreactivity was significantly weaker in mucinous gastric carcinoma than nonmucinous gastric carcinoma (Choi et al., 2010).

Some of the genes such as PTEN, OKL38, CYP2J2 (cytochrome P450 2J2), and AARS (aminoacyl-tRNA synthetases) showed underexpression in the highly peritoneal-metastatic cell line compared to parental cell line in our study. PTEN is a tumor suppressor gene that has many functions, for example, it suppresses apoptosis, promotes cell survival, induces G1 cell cycle arrest, inhibits focal adhesion, spreading, and migration by dephosphorylating focal adhesion kinase, and regulates tumor-induced angiogenesis (Shapira et al., 2004). OKL38, a novel pregnancy-induced growth inhibitory gene, has a growth inhibitory property (Kang et al., 2002). CYP2J2 plays important roles in the metabolism of therapeutic drugs, such as astemizole and ebastine, as well as endogenous fatty acids (Ong et al., 2004). However, these genes related to peritoneal metastasis of gastric cancer are not understood and further investigations are necessary.

In addition, the results of gene chip data required the validation of gene expression using other methods such as RT-PCR, real-time PCR or Northern blotting. When gene chip was applied in the beginning, the quality of microarray was not satisfactory from clone preparation, chip production, variations from hybridization, or inadequate data analysis. However, with improved chip quality and hybridization technique, recent microarray data showed good concordance with RT-PCR or Northern blot method with reliable biological meanings (Lee et al., 2005; Davidson et al., 2006). In the present study, upregulation of the S100A4 and CTNNB1 genes or downregulation of the PTEN gene in GC9811-P were also observed using RT-PCR analysis. We confirmed that the expression levels of the two methods were completely concordant.

Taken together, by using GC9811 and GC9811-P cell lines as a suitable model and screening DNA microarray, we obtained 248 genes that supposedly have potential roles in peritoneal dissemination of gastric adenocarcinoma. Therefore, the data presented here provide important information, and further study of the functions of these genes would be useful for elucidating the molecular mechanisms of gastric cancer with peritoneal metastasis.

\section{ACKNOWLEDGMENTS}

Research supported by grants from the National Natural Science Foundation of China (\#30670969, \#30860332,\#81160308). We also thank Taidong Qiao for excellent technical assistance.

\section{REFERENCES}

Arboleda MJ, Lyons JF and Kabbinavar FF (2003). Overexpression of AKT2/protein kinase Bh leads to up-regulation of h1 integrins, increased invasion, and metastasis of human breast and ovarian cancer cells. Cancer Res. 63: 196-206.

Bai F, Liang J, Wang J, Shi Y, et al. (2007). Inhibitory effects of a specific phage-displayed peptide on high peritoneal metastasis of gastric cancer. J. Mol. Med. 85: 169-180.

Brito M, Malta-Vacas J, Carmona B, Aires C, et al. (2005). Polyglycine expansions in eRF3/GSPT1 are associated with gastric cancer susceptibility. Carcinogenesis 26: 2046-2049.

Cho YG, Nam SW, Kim TY, Kim YS, et al. (2003). Overexpression of S100A4 is closely related to the aggressiveness of gastric cancer. APMIS 111: 539-545.

Choi MG, Sung CO, Noh JH, Kim KM, et al. (2010). Mucinous gastric cancer presents with more advanced tumor stage and weaker beta-catenin expression than nonmucinous cancer. Ann. Surg. Oncol. 17: 3053-3058. 
Davidson B, Zhang Z, Kleinberg L, Li M, et al. (2006). Gene expression signatures differentiate ovarian/peritoneal serous carcinoma from diffuse malignant peritoneal mesothelioma. Clin. Cancer Res. 12: 5944-5950.

Hippo Y, Yashiro M, Ishii M, Taniguchi H, et al. (2001). Differential gene expression profiles of scirrhous gastric cancer cells with high metastatic potential to peritoneum or lymph nodes. Cancer Res. 61: 889-895.

Huerta S, Harris DM, Jazirehi A, Bonavida B, et al. (2003). Gene expression profile of metastatic colon cancer cells resistant to cisplatin-induced apoptosis. Int. J. Oncol. 22: 663-670.

Jemal A, Murray T, Ward E, Samuels A, et al. (2005). Cancer statistics, 2005. CA Cancer J. Clin. 55: 10-30.

Kang YH, Lee HS and Kim WH (2002). Promoter methylation and silencing of PTEN in gastric carcinoma. Lab. Invest. 82: 285-291.

Lazăr D, Raica M, Sporea I, Tăban S, et al. (2006). Tumor angiogenesis in gastric cancer. Rom. J. Morphol. Embryol. 47: 5-13.

Lee SS, Jeong HE, Liu KH, Ryu JY, et al. (2005). Identification and functional characterization of novel CYP2J2 variants: G312R variant causes loss of enzyme catalytic activity. Pharmacogenet. Genomics 15: 105-113.

Li DW, Wu Q, Peng ZH, Yang ZR, et al. (2007). Expression and significance of Notch1 and PTEN in gastric cancer. $A i$ Zheng 26: 1183-1187.

Li J, Wu Y, Qian X and Sha B (2006). Crystal structure of yeast Sis1 peptide-binding fragment and Hsp70 Ssa1 C-terminal complex. Biochem. J. 398: 353-360.

Mori K, Aoyagi K, Ueda T, Danjoh I, et al. (2004). Highly specific marker genes for detecting minimal gastric cancer cells in cytology negative peritoneal washings. Biochem. Biophys. Res. Commun. 313: 931-937.

Motoori M, Takemasa I, Doki Y, Saito S, et al. (2006). Prediction of peritoneal metastasis in advanced gastric cancer by gene expression profiling of the primary site. Eur. J. Cancer 42: 1897-1903.

Ong CK, Ng CY, Leong C, Ng CP, et al. (2004). Genomic structure of human OKL38 gene and its differential expression in kidney carcinogenesis. J. Biol. Chem. 279: 743-754.

Retterspitz MF, Monig SP, Schreckenberg S, Schneider PM, et al. (2010). Expression of \{beta\}-catenin, MUC1 and c-met in diffuse-type gastric carcinomas: correlations with tumour progression and prognosis. Anticancer Res. 30: 4635-4641.

Schena M, Shalon D, Davis RW and Brown PO (1995). Quantitative monitoring of gene expression patterns with a complementary DNA microarray. Science 270: 467-470.

Schieren G, Rumberger B, Klein M, Kreutz C, et al. (2006). Gene profiling of polycystic kidneys. Nephrol. Dial. Transplant 21: 1816-1824.

Shapira M, Ben-Izhak O, Bishara B, Futerman B, et al. (2004). Alterations in the expression of the cell cycle regulatory protein cyclin kinase subunit 1 in colorectal carcinoma. Cancer 100: 1615-1621.

Wang J, Wu K, Bai F, Zhai H, et al. (2006). Celecoxib could reverse the hypoxia-induced Angiopoietin-2 upregulation in gastric cancer. Cancer Lett. 242: 20-27.

Wang YY, Ye ZY, Zhao ZS, Tao HQ, et al. (2010). High-level expression of S100A4 correlates with lymph node metastasis and poor prognosis in patients with gastric cancer. Ann. Surg. Oncol. 17: 89-97.

Yanagihara K, Takigahira M, Tanaka H, Komatsu T, et al. (2005). Development and biological analysis of peritoneal metastasis mouse models for human scirrhous stomach cancer. Cancer Sci. 96: 323-332.

Yonemura Y, Endou Y, Kimura K, Fushida S, et al. (2000). Inverse expression of S100A4 and E-cadherin is associated with metastatic potential in gastric cancer. Clin. Cancer Res. 6: 4234-4242.

Yonemura Y, Endo Y, Obata T and Sasaki T (2007). Recent advances in the treatment of peritoneal dissemination of gastrointestinal cancers by nucleoside antimetabolites. Cancer Sci. 98: 11-18.

Yoon CS, Hyung WJ, Lee JH, Chae YS, et al. (2008). Expression of S100A4, E-cadherin, alpha- and beta-catenin in gastric adenocarcinoma. Hepatogastroenterology 55: 1916-1920.

Yoshikawa T, Yanoma S, Tsuburaya A, Kobayashi O, et al. (2006). Expression of MMP-7 and MT1-MMP in peritoneal dissemination of gastric cancer. Hepatogastroenterology 53: 964-967. 